\section{MedienPädagogik}

www. medienpaed.com
Zeitschrift für

Theorie und Praxis

der Medienbildung

ISSN 1424-3636

Themenheft Nr. 13: Kinderfernsehen wieder zum Thema machen!

\title{
Fernsehen und Internet als konvergierende Wissensinstanzen für Kinder
}

\author{
Lothar Mikos und Claudia Töpper
}

\begin{abstract}
Wissenssendungen, die speziell für Kinder gemacht sind, wie Die Sendung mit der Maus, Löwenzahn, Wissen macht Ah!, Willi will's wissen, und Kindernachrichten wie Logo und Neuneinhalb finden ihr Publikum. Ältere Kinder schauen bereits lieber Wissenssendungen für Erwachsene wie Galileo und Wunderwelt Wissen.

Die Nutzung dieser Sendungen zeigt, dass Kinder ihren Wissensdurst auch mit und beim Fernsehen stillen. Aber was wollen Kinder vom Fernsehen wissen? Welche Wissensbereiche spielen eine Rolle? Wie verbinden die Kinder dabei die Nutzung des Fernsehens und des Internets?

Anhand der Ergebnisse verschiedener empirischer Studien der Autoren aus den letzten Jahren stellt der Beitrag die konvergenten Aspekte von Fernsehen und Internet als Wissensinstanzen für Kinder dar. Dabei wird sowohl auf die Rolle der beiden Medien bei der Informationsbeschaffung und Wissensvermittlung für unterschiedliche Altersgruppen und verschiedene Bildungshintergründe eingegangen wie auf geschlechtsspezifische Unterschiede. Der Beitrag mündet in Konsequenzen für konvergente Angebote zu Bildung und Wissen in Fernsehen und Internet.
\end{abstract}

\section{Einleitung}

Mit der Entwicklung, Verbreitung und Verfügbarkeit neuer Medien hat sich vermutlich auch die Wissensorganisation und -generierung von Kindern und Jugendlichen verändert. Heutzutage stehen ihnen häufig mehrere mediale Möglichkeiten der Informationsbeschaffung zur Verfügung. Das Fernsehen ist jedoch trotz des wachsenden Einflusses neuer Medien nach wie vor Leitmedium und besitzt einen zentralen Stellenwert bei der Konstruktion, Distribution und Rezeption von Wissen.

Kinder stillen ihren Wissensdurst mit und beim Fernsehen. Während die jüngeren Kinder zwischen drei und neun Jahren vor allem die Programme des Kinderfernsehens, insbesondere der auf Kindersendungen spezialisierten Sender Ki.Ka und Super RTL sehen, bevorzugen die 10- bis 13-Jährigen dagegen Programme des «Erwachsenenfernsehens» (vgl. Feierabend / Klingler 2006: 145 f.).

Wissenssendungen, die speziell für Kinder gemacht sind, wie Die Sendung mit der Maus, Löwenzahn, Wissen macht Ah!, Willi will's wissen oder Kindernachrichten wie Logo und Neuneinhalb finden ihr Publikum. Ältere Kinder und Jugendliche schauen bereits lieber die Wissenssendungen für Erwachsene wie Galileo oder Wunderwelt Wissen. 
Aber was wollen die Kinder durch die Fernsehsendungen eigentlich genau erfahren? Welche Wissensbereiche spielen für sie eine Rolle? Und wie verbinden sie dabei die Nutzung des Fernsehens und des Internets als Informationsmedien? Im Folgenden werden Ergebnisse einer empirischen Studie vorgestellt, die sich mit dem Aspekt der konvergenten Nutzung von Fernsehen und Internet als Wissensinstanzen für Kinder beschäftigt hat.

\section{Methodische Vorgehensweise}

Die Studie wurde im August und September 2005 im Auftrag des Westdeutschen Rundfunks durchgeführt. Ziel war es, thematische Interessen und Wissensbedürfnisse von Kindern sowie die Rolle des Fernsehens und des Internets bei der Aneignung von Wissen ${ }^{1}$ zu untersuchen. Die Studie wurde begleitend zur Aktion «Frag doch mal ...» durchgeführt, bei der die Redaktion der Sendung mit der Maus im Jahr 2005 Kinder aufforderte ihre Fragen an den WDR zu schicken. Insgesamt folgten knapp 76.000 Kinder dem Aufruf und schickten ihre Fragen an den Sender. Die am häufigsten gestellte Frage war: «Warum ist der Himmel blau?». Sie wurde allerdings lediglich von 781 Kindern (1,03 Prozent) gestellt, was zeigt, wie breit das Spektrum der Fragen und der Wissensbedürfnisse der Kinder ist. Es folgten Fragen rund um die Sendung mit der Maus auf Platz zwei, «Wie kommt der Regenbogen an den Himmel?» auf Platz drei, «Wie funktioniert ein Fernseher? / Wie kommt das Bild bzw. die Maus in den Fernseher?» auf Platz vier, und «Wie fliegt ein Flugzeug?» auf Platz fünf.

Die wissenschaftliche Begleitstudie sollte weiteren Aufschluss darüber geben, welche Themengebiete die Kinder interessieren und welche Rolle das Fernsehen und das Internet - neben anderen Wissensinstanzen wie Eltern und Schule - bei der Aneignung von Wissen spielen. Insgesamt wurden dafür zehn themenfokussierte Gruppendiskussionen mit 61 Kindern im Alter von sechs bis 13 Jahren durchgeführt. Dabei handelte es sich jeweils um fünf Gruppen mit 6- bis 9-Jährigen und 10- bis 13-Jährigen. Die Gruppen bestanden in der Regel aus mindestens vier bzw. maximal sechs Kindern. Vorzugsweise wurden die Kinder in einer ihnen vertrauten Umgebung (vor allem Horte und Kinderfreizeitstätten) aufgesucht und dort von einem Moderator oder einer Moderatorin befragt. Die Befragung von Realgruppen wurde entsprechend dem in der qualitativen Forschung geltenden Prinzip der Naturalistizität als Vorteil gewertet, da gruppendynamische Aushand-

Die Begriffe Wissen und Information werden im Sinne der Definition von Rainer Kuhlen gebraucht. Dieser definiert Wissen als die «zu einem bestimmten Zeitpunkt durch Anwendung bestimmter Verfahren (Erfahrung, Experimente, Logik, Kommunikation) gewonnene und als gültig angesehene Menge der Aussagen über Objekte und Sachverhalte der Welt [...]. Information wird aus bestehendem Wissen erarbeitet. Wissen wird zur Information nur durch den Bezug auf einen aktuellen Handlungszusammenhang (Information ist Wissen in Aktion).» (Kuhlen 2000: 2) 
lungsprozesse bei vertrauten Personen in der Regel minimal sind und die Gruppenmitglieder über gemeinsame Erfahrungshorizonte verfügen.

Um regionale Unterschiede berücksichtigen zu können, fanden je zwei Gruppendiskussionen in Grossstädten (Berlin und München), einer mittelgrossen Stadt (Erfurt) und zwei ländlichen Regionen (westliches Niedersachsen und Bergisches Land) statt. Die befragten Kinder unterschieden sich zusätzlich durch verschiedene Bildungsgrade. Da soziodemografische Variablen wahrscheinlich einen starken Einfluss auf die Bildungschance und das dadurch erreichbare Bildungsniveau haben, wurden Interviews mit Kindern aus Stadtteilen mit einer hohen Konzentration sozial schwacher Bewohner durchgeführt sowie mit Kindern aus Stadtteilen mit entsprechend besser situierten Bewohnern². Ausgangspunkte zur Erfassung medienübergreifender Nutzungsweisen und Nutzungsmotiven von Internet und Fernsehen boten sich nach unserer Ansicht über Fragen nach favorisierten Tätigkeiten und Vorlieben der Kinder. Über mediale Angebote befriedigen sie zahlreiche Interessen und Wissensbegierden. Im Mittelpunkt unserer Untersuchung stand die Frage, welche Nutzungswege die Kinder dabei verfolgen und inwiefern sie von konvergenten Angeboten Gebrauch machen. Heute gibt es nur noch selten mediale Einzelerzeugnisse, sondern eher Produktverbünde. Auch zu Wissenssendungen finden sich DVDs, Bücher oder Internetangebote. Innerhalb und zwischen den einzelnen Medien bestehen entsprechende Verweisstrukturen, die die Nutzer auf die unterschiedlichen Angebote aufmerksam machen sollen. In Bezug auf den Wissenserwerb interessierte uns hierbei, welche Wege die Kinder innerhalb der Produktverbünde einschlagen, wenn sie etwas wissen wollen: wen fragen sie und welche Medien nutzen sie? Unter medienkonvergenten Nutzungswegen verstehen wir in diesem Kontext demnach Nutzungsstrukturen von Kindern, die über Einzelmedien hinausgehen.

Anhand eines strukturierten Leitfadens wurden dementsprechend insgesamt drei Themenbereiche angesprochen: Interessensgebiete, Informationsverhalten und die Nutzung konvergenter Angebote, Wahrnehmung und Bewertung von (konvergenten) Angeboten der Wissensvermittlung insbesondere durch das Fernsehen und das Internet.

Nach Beendigung der Diskussion wurden die Kinder aufgefordert, einen einseitigen Ergänzungsfragebogen auszufüllen, der die soziodemografischen und soziokulturellen Daten sowie ihre individuelle Mediennutzung und ihre Medienpräferenzen erfassen sollte. Kinder, die noch nicht ausreichend gut genug lesen und schreiben konnten wurden beim Ausfüllen von den Moderatoren oder Moderatorinnen unterstützt.

2 Der Bildungsgrad der Kinder wird dabei nicht exakt beschrieben, wurde jedoch für die empirische Arbeit als ausreichend betrachtet. 
Die Gruppendiskussionen wurden mit einem Tonbandgerät aufgezeichnet und anschliessend transkribiert. Die Auswertung erfolgte themen- und strukturanalytisch und die Originalzitate wurden bei der Interpretation der Ergebnisse und der Darstellung beispielhaft einbezogen.

Die Studie hat explorativen Charakter. Sie war nicht auf Repräsentativität angelegt, sondern darauf, möglichst tief in die Wissensbedürfnisse, den Wissenserwerb, die Mediennutzung und den Alltag der Kinder einzudringen.

\section{Was wollen Kinder wissen?}

Die Interessen der Kinder sind sehr vielfältig. Sie lassen sich jedoch grob drei Bereichen zuordnen: (1) Die Welt begreifen, (2) Freizeit und Konsum, (3) sozialer Umgang.

\section{Die Welt begreifen}

Im Alltag begegnen den Kindern viele Dinge, die sie (noch) nicht begreifen können. Das Interesse für Alltagsfragen ist daher besonders gross. Die befragten Kinder haben ein starkes Bedürfnis nach deklarativem und prozeduralem Wissen. Sie wollen die Welt erklärt bekommen und wissen, wie Dinge funktionieren. Ein besonderes Interesse an Technik, und hier insbesondere an den Funktionsweisen und der Herstellung von Medientechnik haben dabei vor allem die Jungen. Computer und Computerspiele, Fernsehen, DVDs und Handys stehen hoch im Kurs. Daneben möchten die Kinder auch wissen, wie der menschliche Körper funktioniert.

Von besonderem Interesse sind auch Tiere. Die Kinder sind vor allem neugierig darauf, wie sie leben, wie sie entstanden sind, welche Besonderheiten sie haben und wie man mit ihnen umgeht. Dabei handelt es sich vorrangig um Tiere der heimischen Fauna, welche ihnen am ehesten im alltäglichen Leben begegnen - auch weil einige von ihnen als Haustiere in Frage kommen. Exotische Tiere spielen erst danach eine Rolle.

Religion und Politik im engeren Sinne werden kaum thematisiert, während historische Themen vor allem auf jüngere Kinder eine grosse Faszination ausüben. Sie interessieren sich hauptsächlich für die beiden Weltkriege. Ausserdem wüssten die jüngeren Kinder im Alter zwischen sechs und neun Jahren gerne mehr über die Entstehungsgeschichte der Menschen, über die Steinzeit und über die alten Ägypter, Griechen, Römer bis hin zur Entdeckung Amerikas.

Neben den mehr oder weniger existentiellen Fragen des (Alltags-)Lebens entwickeln die Kinder in einer immer komplexer werdenden Welt ein grosses Bedürfnis nach Orientierungswissen. Die Kinder in unserer Studie waren noch sehr beeindruckt vom Tsunami im indischen Ozean am 26.12.2004, von dem sie in erster Linie über das Fernsehen erfahren hatten. Sie wollen wissen, wie solche Naturkatastrophen entstehen und wie die betroffenen Menschen damit umgehen. Die 11jährige Nanette aus Berlin beispielsweise interessiert, wie Tsunami-Warnsysteme 
funktionieren: «Weil man fühlt sich dann irgendwie sicherer, wenn man weiss, wie das funktioniert, 》 und die ebenfalls 11-jährige Katharina interessiert sich für Tornados oder die Eiszeit, weil sie Angst davor hat, «dass die so kommt oder so. Weil das mit der Umweltverschmutzung und mit der globalen Erwärmung und so was.» Das Wissen über Funktionsweisen wird von den Kindern als Problemlösungsstrategie genutzt, um mit ihren Ängsten umzugehen. In diesem Zusammenhang zeigt sich ein überraschender Effekt: Umwelt ist für die Kinder ein eher angstbesetztes Thema, das vor allem mit Naturkatastrophen in Verbindung gebracht wird. Umweltschutz im positiven Sinne spielt bei den Kindern in der Studie keine Rolle.

\section{Freizeit und Konsum}

Auch das Interesse für verschiedene Sportarten ist vielfältig. Lediglich die Jungen haben besondere Präferenzen. Sie interessieren sich vor allem für Profifussball. Daneben sehen sie sich noch gerne Wrestling an.

Computer- und Konsolenspiele werden unabhängig von Alter und Geschlecht genutzt. Die Mädchen spielen eher Spiele wie Der kleine Eisbär, während sich die Jungen für sogenannte Egoshooter und Sportspiele (Formel 1, World Race, FIFA 2005) begeistern können. Bücher werden von allen befragten Kindern gerne gelesen.

Mode ist besonders für jüngere Mädchen ein Thema, während bei einigen der befragten Jungen besondere Mode-Marken eine Rolle spielen.

\section{Sozialer Umgang}

Zwei Themen stehen für die Kinder in diesem Bereich an erster Stelle: Liebe und Konflikte. In beiden Fällen geht es um Beziehungsgestaltungen zu anderen Kindern, positive wie negative. Liebe ist für die jüngeren Kinder eher ein peinliches Thema, sie interessieren sich vorrangig dafür, wie das jeweils andere Geschlecht «tickt» und wie man sich am besten kennen lernen kann. Für die älteren Kinder ist das Thema «Sexualität» von Bedeutung. Sie wollen wissen, «wie das geht» und welche Verhütungsmittel es gibt. Fragen zu Aids, Verhütung, Kindesmissbrauch und Schutz vor sexuellen Übergriffen bewegen sie dabei am meisten.

Wichtig ist den Kindern auch zu erfahren, wie man Konflikte und Probleme löst. Zwar haben sie hier teilweise schon individuelle Strategien für spezifische Konflikte wie Streit mit Freunden entwickelt, doch stehen sie gewissermassen täglich vor neuen Herausforderungen, für die sie noch keine Problemlösungskompetenz besitzen.

\section{Wissensinstanzen}

Eltern

Für die Kinder sind verschiedene Wissensinstanzen von Bedeutung, denen sie spezifische Fähigkeiten zuschreiben. Die wichtigste Wissensinstanz und die ersten 
Ansprechpartner sind die Eltern. Die 9-jährige Layla meint dazu: «Ja, ich glaub, wenn ich ein Problem hab, dann wende ich mich an meine Eltern und wenn ich ein Problem mit meinen Eltern hab, dann frag ich meine Freunde.» Hier zeigt sich bereits, dass Kinder recht früh wissen, welche der ihnen zur Verfügung stehenden Wissensinstanzen für welche Fragen oder Probleme in Frage kommen. Mütter und Väter werden zu unterschiedlichen Themengebieten befragt. Während die Mütter bei Themen wie "Sprache», «Liebe» beziehungsweise «Verliebtsein» gefragt sind, liegen die Kompetenzen der Väter nach Ansicht der Kids in den Bereichen Computer und Internet, Mathematik und Technik sowie Sport. Auf die Frage, ob sie ihrem Vater andere Fragen stellen würde als ihrer Mutter antwortet die 9-jährige Magdalena: «Ja, zum Beispiel über Sport oder über Fussball, da wissen ja die Frauen nicht Bescheid. Oder zum Beispiel bei Formel 1 oder so was, da wissen die gar nicht Bescheid.»

Allerdings weichen die Geschlechtergrenzen in Bezug auf Computerprobleme auf, denn hier werden vereinzelt auch die Mütter als Expertinnen genannt.

\section{Grosseltern}

Die Grosseltern sind in erster Linie für geschichtliche Fragen von Belang und hier besonders hinsichtlich ihrer Erlebnisse im Zweiten Weltkrieg und im Zusammenhang mit Auswanderung beziehungsweise Immigration. Die 9-jährige Marlene interessiert sich für die Weltkriege, «weil ich ,ne Uroma hab. Und mein anderer Opa, der hat auch ganz, ganz viel erlebt.»

Geschwister und Freunde werden vor allem bei Konflikten im Freundeskreis um Rat gefragt. Von ihnen wird vermutet, dass sie auf diesem Gebiet Erfahrungen haben.

\section{Fernsehen}

Während die Eltern und Grosseltern als erste Anlaufstation für gezielte Fragen gelten, befriedigt das Fernsehen eher das Bedürfnis nach einer Art Überblickswissen. Da Eltern und Grosseltern den Kindern nicht alle Fragen beantworten können, wird dem Fernsehen eine grosse Bedeutung beigemessen, vor allem den klassischen Wissenssendungen. Durch das Fernsehen kann man viel lernen, meint die 9-jährige Marita, vor allem, «wenn man Wissenssendungen anschaut, dann schon. Bei Witzsendungen eher weniger. [...] Im Fernseher da will man halt alles richtig interessant machen und da erklärt man viel mehr drum herum und die Eltern wollen es halt kurz machen, bei mir jedenfalls oft, weil sie dann noch was zu tun haben.» Die 9-jährige Melanie hat eine klare Empfehlung für Erwachsene: «Das können ruhig ab und zu auch mal Erwachsene schauen, wenn sie es unbedingt wollen. Wen sollen sie sonst fragen? Den Gott? Der kann die Fragen ja nicht beantworten. Da kann man ruhig mal Die Sendung mit der Maus gucken.» Und der ebenfalls 9-jährige Tom meint: «Wenn ich was wissen will? Wenn ich Glück hab, kommt es 
im Fernsehen, wenn nicht, frag ich meine Mutter.» Hier zeigt sich erneut, dass Kinder genau wissen, von welcher Wissensinstanz sie was erwarten können. Zugleich scheinen sie interne Hierarchien von Wissensinstanzen zu bilden, die ihnen erlauben auch Alternativen in Betracht zu ziehen - in diesem Fall die Mutter als Alternative zum Fernsehen, auch wenn sie in der Rangfolge hinter dem Fernsehen zu rangieren scheint.

Die Kinder weisen dem Medium Fernsehen differenzierte Funktionen zu. In Bezug auf den Wissenserwerb ist ihnen bewusst, was das Medium leisten kann und was nicht, oder wie es die 9-jährige Julia formuliert: «Mathe kann man nicht im Fernsehen lernen.» Wissen wird durch das Fernsehen eher zufällig und flüchtig erworben. Zur gezielten Informationssuche erscheint es den Kindern ungeeignet. So antwortet der 13-jährige Klaus auf die Frage, warum man im Fernsehen nicht so viel lernen kann: «Da kann man ja die Sender nicht bestimmen, was jetzt kommen soll. Das steht ja alles im Videotext oder der Fernsehzeitung.»

Die Rezeption von Fernsehsendungen dient den Kindern anscheinend eher zum Erwerb von Überblickswissen, das ihnen zahlreiche thematische Anregungen bietet. Die 12-jährige Caro beispielsweise sieht sich gerne die Telenovela Verliebt in Berlin an und sie «würde interessieren, wie Designer Kleider machen. Weil ich gucke auch Verliebt in Berlin und da sind so Designer in der Serie und wie die das organisieren, dass da jetzt einer die Stoffe holt, oder dass der das persönlich machen tut, und das würde ich gern wissen.» Die für die Kinder interessanten Anregungen aus den Fernsehsendungen werden jedoch nur selten von anderen Wissensinstanzen aufgegriffen. So spielt auch die Schule als Wissensinstanz überraschenderweise in diesem Zusammenhang für die Kinder kaum eine Rolle - mit Ausnahme der befragten Kinder aus Bayern, die der Schule einen gewissen Stellenwert bei der Wissensvermittlung bescheinigen.

\section{Internet}

Im Gegensatz zum Fernsehen bietet das Internet den Kindern die Möglichkeit gezielt nach Informationen zu suchen. Die Vor- und Nachteile der beiden Medien werden von den von uns befragten Kindern pragmatisch eingeschätzt. Alle Kinder kennen das Internet als Möglichkeit schnell Daten und Fakten zu recherchieren und gezielt Informationen zu nutzen. Die etwas älteren Kinder nutzen es dabei bereits selbst oder - vor allem im Fall der jüngeren Kinder - lassen sich von Geschwistern und Eltern die gesuchten Informationen beschaffen. Die Kinder schreiben dem Internet jedoch nicht nur informationsorientierte Nutzungsmöglichkeiten zu, sondern es dient ihnen auch für spielorientierte Tätigkeiten oder zur Kommunikation. Die von vielen Kindern bevorzugte Kommunikationsform im Internet ist der Chat. Der kommunikative Austausch innerhalb der Chat-Gemeinschaften dient vor allem dem Erwerb von Wissen über das andere Geschlecht und dem sozialen Umgang miteinander, wie die Aussage des 11-jährigen Ditmir zeigt: «Ja, wenn ich Fragen 
hätte zu Mädchen, dann wäre ich auch Chatten gegangen, im Computer, Internet.» Im Vergleich zur Nutzung des Internets als Recherchemittel für die Schule oder bestimmte Interessensgebiete, ist beim Chatten der Wissenszuwachs im klassischen Sinn eher gering. Es wird weniger deklaratives Faktenwissen gesammelt, sondern eher prozedurales Wissen. Die Kinder setzen sich dynamisch und kreativ mit ihrer eigenen Identität auseinander und erproben Formen des sozialen Umgangs mit dem anderen Geschlecht.

Insgesamt schreiben alle befragten Kinder dem Internet wissensbezogene Prioritäten zu und benutzen es teilweise zur Recherche für die Schule oder aus Interesse an bestimmten Fragestellungen, häufig gemeinsam mit den Eltern. Gleichzeitig suchen sie im Netz aber auch nach Informationen über Konsumartikel und Lebensstile, spielen im Internet oder Chatten. Informationsrecherche im Internet bedeutet für die Kinder nicht nur Akkumulation von Faktenwissen, sondern auch Orientierung über symbolische Ressourcen zur Selbstpositionierung. Dabei unterscheiden die Kinder nicht zwischen Information und Unterhaltung (vgl. hierzu auch Kaiser / Mikos / Töpper 2006). Offenbar gilt hier auch für die Kinder, was Dehm und Storll für Erwachsene festgestellt haben: «Unterhaltung und Information sind kein Gegensatz» (vgl. Dehm / Storll 2002: 16).

\section{Konvergente Aspekte von Fernsehen und Internet als Wissensinstanzen für Kinder}

Fernsehen kann für alle befragten Kinder als das zentrale Ausgangsmedium und Verweisgeber auf andere Informationsangebote betrachtet werden. Eine konvergente Nutzung des Fernsehens und des Internets ist dabei primär themenabhängig und über spezifische Interessen der Kinder motiviert. Zum einen werden Fernsehen und Internet komplementär genutzt und zum anderen dient das Internet als Substitutionsang ebot für Themenbereiche, die nach Meinung der befragten Kinder durch das Fernsehangebot vernachlässigt werden.

\section{Komplementäre Nutzung von Fernsehen und Internet}

Vor allem für den Bereich Freizeit und Konsum, also die Themenbereiche Sport, Musik, Computer- und Konsolenspiele werden Internet und Fernsehen komplementär genutzt. Über aktuelle Musikangebote informieren sich die befragten Kinder beispielsweise zunächst im Fernsehen, um sich dann die gewünschten Songs im Internet herunterzuladen.

\begin{tabular}{|c|c|}
\hline l: & Wie informiert ihr euch denn über Musik? \\
\hline Micha (11 Jahre): & Im Fernsehen. \\
\hline I: & Und was guckt ihr da so? \\
\hline Caro (12 Jahre): & Viva. \\
\hline Alle: & Ja. \\
\hline Micha: & Ja, und MTV. \\
\hline
\end{tabular}


I: $\quad$ Informiert ihr euch auch manchmal im Internet?

Micha: Hab mir schon viel runtergeladen. Hip Hop und so.

Auch spezielle Internetangebote von Fernsehsendungen werden genutzt. Zum Beispiel laden sich einige der befragten Kinder Bastelanleitungen der Sendungen Art Attack und Fingertipps aus dem Internet herunter. Die 12-jährige Caro findet Fingertipps am besten, weil: «Das kann man auch im Internet ausdrucken, wie das geht und das kann man dann auch selbst machen.» Die Einbindung des medialen Angebots in den lebensweltlichen Kontext der Kinder ist wie bei den Wissensgebieten, die die Kinder interessieren, auch bei der konvergenten Mediennutzung von Bedeutung. Eine konvergente Nutzung von Fernsehsendungen und den entsprechenden Internetangeboten scheint eher zu erfolgen, sobald Anknüpfungspunkte zur Übernahme und zur Relevanz der Informationen für den eigenen Lebensalltag der Kinder vorhanden sind.

Eine komplementäre Nutzung der beiden Medien Fernsehen und Internet bietet den Kindern die Möglichkeit Informationen aus beiden Medien zu kombinieren, zu ergänzen, zu vertiefen oder weiterzuführen und ihr Wissen in bestimmten Interessensgebieten anzureichern. Die konvergente Nutzung wird vor allem durch spezifische persönliche Interessen und durch den Bezug auf die eigene Lebenswirklichkeit der Kinder geprägt.

\section{Substitutionstendenzen}

Trotz vielfältiger Anregungen durch Fernsehsendungen, werden manche Themen nach Meinung der Kinder durch das Fernsehen nicht ausreichend verhandelt. Vor allem in dem Bereich «Sozialer Umgang» fehlen den Kindern entsprechende Fernsehangebote, die für sie relevante Themen wie «Liebe und Sexualität» aufbereiten. Informationen zu diesem Bereich suchen sich die Kinder gezielt in anderen Medien. Da Fragen zum Thema «Sexualität» häufig weder von den Eltern noch von der Schule ausreichend beantwortet werden und auch im Fernsehen die sie interessierenden Fragen zu Aids, Verhütung, Kindesmissbrauch und Schutz vor sexuellen Übergriffen nicht genügend abgedeckt werden, ist die Jugendzeitschrift Bravo Hauptinformationsquelle für diesen Bereich.

Wissen über das andere Geschlecht und Formen des sozialen Umgangs mit Gleichaltrigen erwerben die Kinder aber nicht nur durch die Bravo, sondern auch durch spezifische Internetangebote. Vor allem die von den Kindern im Internet beliebte Kommunikationsform Chatten dient ihnen in diesem Fall als Substitutionsangebot für fehlende (Kinder-)Fernsehsendungen, die Themen dieser Art altersgerecht aufbereiten. 


\section{Unterschiedliche Nutzungsformen}

Innerhalb der Gruppendiskussionen mit den Kindern liessen sich in Bezug auf eine konvergente Mediennutzung unterschiedliche Nutzertypen feststellen, die in ihren Aktivitätsniveaus variieren und sich als eher informationsorientiert versus konsumorientiert beschreiben lassen. Unbestritten nutzen alle befragten Kinder Fernsehen und Internet vor allem für unterhaltungs- und spielorientierte Tätigkeiten. Daneben unterscheiden sich jedoch diejenigen Kinder, die in ihrer Freizeit aktiv spezifische Interessen verfolgen in ihrer konvergenten Mediennutzung von denjenigen Kindern, die sich als eher passiv beschreiben lassen. Die Kinder unserer Befragung, die in einem eher niedrigeren Bildungsniveau aufwachsen, suchen im Internet weniger gezielt nach Informationen. Auf die Frage, was er denn mache, wenn er etwas Bestimmtes wissen wolle, antwortet der 12-jährige Arber: «Da musst du Satelliten-System draufmachen und alles durchsuchen». Die befragten Kinder wissen zwar, was welche Medien leisten können, Metawissen über den Wissenserwerb selbst ist allerdings scheinbar nur unzureichend vorhanden. Sie lassen sich lieber durch (Fernseh-)Werbung leiten und nutzen die vom Markt offerierten Internetangebote, die für sie bequem zu erreichen sind. Ihr Interesse gilt eher den leicht zugänglichen und konsumierbaren Bestandteilen der konvergenten Medienwelt.

Die befragten Kinder aus höheren Bildungsniveaus dagegen verfolgen häufiger aktiv spezifische Interessen, wie beispielsweise die Ausübung von sportlichen Aktivitäten in einem Verein, das Erlernen eines Musikinstruments oder ein besonderes Interesse an Natur und Technik. Diese Vorlieben werden von den Kindern nicht nur in ihrer Freizeit aktiv ausgeübt, sondern sie informieren sich darüber auch durch die Nutzung unterschiedlicher Medien. Sie suchen - scheinbar auch angeregt durch ihr soziales Umfeld, dabei vor allem die Eltern, die ihnen helfen - gezielt nach Möglichkeiten ihr Wissen in diesen Bereichen anzureichern. Besondere Interessensgebiete werden daher auf einschlägigen Internetseiten und durch ausgewählte Fernsehsendungen verfolgt. Der 9-jährige David beispielsweise möchte Vulkanologe werden, weil er «das mal in Galileo geguckt [hat]» und ihn das von Anfang an interessiert hat. Deshalb möchte er auch gerne einmal in seinem Leben nach Lanzarote: "Das ist [sein] Lebenstraum.» Auf die Frage, was er mache, wenn er etwas mehr über sein spezifisches Interesse an Vulkanen erfahren wolle, antwortet David - im Gegensatz zu Arber - : «lch hab ein Buch und da guck ich dann nach oder ich guck mal im Internet, aber mit meiner Mama, weil ich kann das noch nicht so gut.»

\section{Fernsehen und Internet in der politischen Bildung Jugendlicher}

Diese Unterschiede zwischen Kindern und Jugendlichen aus «bildungsnahen» und «bildungsfernen» Milieus zeigten sich auch in einer anderen Studie, die von den Autoren durchgeführt wurde (vgl. Töpper/Mikos 2006). Im Auftrag der Bundes- 
zentrale für politische Bildung wurden Gruppendiskussionen mit insgesamt 60 Jugendlichen bzw. jungen Erwachsenen zwischen 14 und 25 Jahren vorwiegend aus «bildungsfernen» Milieus zu den Möglichkeiten medial vermittelter politischer Bildung durchgeführt. In Bezug auf die Konvergenz von Fernsehen und Internet zeigte sich, dass die Jugendlichen zwar Zugang zu dem Medium haben, es aber anscheinend anders nutzen als formal besser gebildete Jugendliche.

Obwohl sie es allgemein wichtig erachten, politisch informiert zu sein, beziehen, die in den Gruppendiskussionen befragten Jugendlichen politische Informationen, wenn überhaupt, vor allem aus Fernsehsendungen. Nachrichtensendungen werden jedoch nur sporadisch, zufällig oder anlassbezogen rezipiert. Vorzugsweise sehen sich die Jugendlichen die Nachrichten auf ProSieben und RTL an. Ihr Informationsverständnis reicht dabei von seriösen Nachrichten bis zu Klatsch und Tratsch, orientiert sich jedoch eher an letzterem. So betrachten sie teilweise auch das Boulevardmagazin taff als Nachrichtensendung.

Das Internet spielt bei den befragten Jugendlichen aus «bildungsfernen» Milieus als Informationsmedium eine untergeordnete Rolle und wird vor allem zur Unterhaltung genutzt. Dies bedeutet jedoch nicht, dass die Jugendlichen das Internet als Möglichkeit sich politisch zu informieren, ablehnen. Im Gegenteil sie stehen dem Medium aufgeschlossen gegenüber, wünschen sich Informationen aber einfach entsprechend ihrer kognitiven Fähigkeiten aufbereitet.

Eine konvergente Nutzung des Fernsehens und des Internets ist auch bei den Jugendlichen abhängig von spezifischen persönlichen Interessen, aber auch von der individuellen Beurteilung der Fernsehsendung, wie der 22-jährige Sascha feststellt: «Wenn einem die Sendung nicht gefällt, warum sollte man dann auf die Seite gehen, die in der Sendung erwähnt wird.» Die durch Fernsehsendungen hervorgerufenen Anreize spezifische auf das Programm abgestimmte Internetseiten zu besuchen, müssen also dementsprechend stark sein oder die Thematik muss die Jugendlichen ausserordentlich interessieren, damit sie entsprechende Internetseiten tatsächlich nutzen. Anreize in Fernsehsendungen können beispielsweise starke Moderationsfiguren oder auch Gewinnspiele und die Möglichkeit eines Downloads der Sendung, beziehungsweise Teile der Sendung. Für die aktive und parallele Nutzung des Fernsehens und Internets entscheidend bleiben jedoch vor allem Thema und Machart der Fernsehsendung.

Trotz der Schwierigkeiten die Jugendlichen zu erreichen, bietet das Internet die Chance zur Förderung politischer Handlungsfelder. Immer wieder betonen die befragten Jugendlichen, unabhängig vom Bildungsgrad, ihr mangelndes politisches (Grund-)Wissen. Das Internet bietet die Möglichkeit (spielerisch) an demokratischen Prozessen teilzunehmen und dadurch nötiges Wissen und Kompetenzen zu vermitteln, die für das Leben in einer globalen Wissensgesellschaft notwendig sind. Allein im Internet funktioniert politische Bildung jedoch nicht. Zusätzlich müssen Anreize durch andere Medien geschaffen werden. Die Hemmschwellen 
zur Nutzung des Mediums müssten ebenso überwunden werden wie die, sich intensiver mit Politik zu befassen - und sich generell mit Wissen aus dem Internet zu versorgen.

Die unterschiedliche Nutzung von Fernsehen und Internet durch Kinder aus verschiedenen Bildungsmilieus, die mit informations- versus konsumorientiert bezeichnet wurde, scheint sich im Jugendalter zu verfestigen. Es zeigt sich, dass die modernen Informations- und Kommunikationstechniken mit ihrem Beitrag zur so genannten Wissensgesellschaft vor allem zur Stabilisierung von Wissensformen und Deutungsmustern herrschender Milieus beitragen (vgl. Bittlingmayer 2005, S. 332). Zugleich scheinen sich biographische Muster der (konvergenten) Mediennutzung, die in der Kindheit ausgebildet werden, in der Jugendphase fortzusetzen. Die aktive Beschaffung von Information und deren Anwendung im Alltag kann offenbar nicht früh genug gelernt werden.

\section{Konsequenzen}

Fernsehen regt Kinder an, sich mit zahlreichen Themen zu beschäftigen. Leider werden diese Themen - von den Erklärungen zu alltagspraktischen Dingen, der Funktionsweise von Geräten oder dem menschlichen Körper über Freizeit- und Konsumthemen bis zu Fragen, die den sozialen Umgang betreffen - offenbar nur unzureichend von anderen Institutionen wie dem Elternhaus und noch weniger der Schule aufgegriffen. In gewissem Sinne kann man davon sprechen, dass das Fernsehen für die Kinder als eine Art Wissensmaschine fungiert, die sie allerdings lediglich mit Fragmenten versorgt und sie damit in erster Linie neugierig auf Themen macht. Tiefgehenderes erfahren sie nur dann, wenn Wissenssendungen, soziales Umfeld oder Schulunterricht zufällig eines dieser Themen aufgreifen und so den Wissensdurst der Kinder stillen können. Insbesondere Kindern aus geringeren Bildungsmilieus fehlen anscheinend entsprechende Anregungen aus dem sozialen Umfeld, um durch (Kinder-)Fernsehen erworbenes Wissen oder Anregungen sinnvoll in ihren lebensweltlichen Kontext zu integrieren. So erzählt der 9-jährige Hassan darüber, wie er sich informiert, wenn er etwas wissen möchte: «lch gucke einfach Wissen macht Ah! oder Galileo. Letztens haben die gezeigt, wie man sich selber Kleber macht. Hab ich schon wieder vergessen. Und die haben gezeigt, wie man Kuchen macht. Ich hab es nicht nachgemacht, weil, da gibt es Zutaten von denen ich gar nicht weiss, wo man die herholt.»

Fehlt eine Einbindung des vermittelten Wissens in den Lebensalltag der Kinder scheint das Fernsehen bei den befragten Kindern eher unspezifisches Wissen zu generieren. Zwar stellt das (Kinder-)Fernsehen vielfältige Informationen zur Verfügung, aber erst die Einbettung dieser Inhalte in Alltagskommunikation führen zur Konstruktion von Wissen. Manchen der befragten Kinder mangelt es in ihrer sozialen Umwelt an Anknüpfungspunkten, um die durch das Fernsehen vermittelten Anregungen einzuordnen und anzuwenden. Der Umgang mit den durch das 
Fernsehen vermittelten Informationen ist dann eher provisorisch, wenig verankert und kann nicht in Wissen übergehen.

Aufgabe der Medienpädagogik ist es daher, das durch Medien vermittelte Wissen gelingend zu integrieren. Dies bedeutet einerseits die bestehenden Tendenzen konvergenter Mediennutzung und informeller Aneignungsstrukturen gezielt zu fördern und andererseits Kindern, die eher konsumorientiert sind und denen es an entsprechendem Metawissen zum Wissenserwerb mangelt, Möglichkeiten einer zielgerichteten und aktiven konvergenten Mediennutzung aufzuzeigen. Dazu bedarf es nicht nur einer Konvergenz der Medien, sondern vor allem einer Konvergenz der Sozialisationsinstanzen, die komplementär zur Wissensgenerierung der Kinder beitragen müssen. Die «Wissensmaschine» Fernsehen muss von Eltern und Schule mindestens ebenso ernst genommen werden, wie die Kinder sie im Rahmen der Peers nehmen. Nur dann können sie auch als vertiefende Ergänzung und Korrektiv von den Kindern wahrgenommen und angenommen werden. Das aber ist, wie das Beispiel des Wissens der Kinder über das Themengebiet Umwelt zeigt, dringend notwendig, um die durch skandalisierende Berichterstattung ausgelösten Ängste, die mit Umwelt verbunden werden, zu bearbeiten. Das Fernsehen muss sich nicht nur in den Kindersendungen, sondern in allen Tagesprogrammen zwar der Verantwortung für die Bildung von Kindern und Jugendlichen bewusst sein, doch muss es sich auch auf die anderen Sozialisationsinstanzen verlassen können, die ebenfalls ihren Beitrag zur Bildung leisten müssen.

Die Ergebnisse der Studien haben gezeigt, dass Kinder bereits sehr früh Vorstellungen davon ausbilden, welches Wissen sie von welchen Wissensinstanzen erwarten können, und auch, was sie nicht erwarten können, wie es bei den Jugendlichen aus «bildungsfernen» Milieus deutlich wurde. Mit konvergenten Inhalten von Fernsehen und Internet lassen sich vor allem die «bildungsnahen» Kinder und Jugendlichen erreichen. Für die «Bildungsfernen» müssen spezielle Formen und Inhalte entwickelt werden, die eher spielerischen Charakter haben. Nur so sind sie für medial vermittelte Bildungsprozesse zu gewinnen.

\section{Literatur}

Bittlingsmayer, Uwe H. «Wissensgesellschaft» als Wille und Vorstellung. Konstanz: UVK, 2005.

Dehm, Ursula; Storll, Dieter. «Spass oder Anspruch: Was erwarten die Zuschauer von guter Fernsehunterhaltung?» Planung \& Analyse, 5 (2002): 16-20.

Feierabend, Sabine; Klingler, Walter. «Was Kinder sehen. Eine Analyse der Fernsehnutzung Drei- bis 13-Jähriger 2005» Media Perspektiven, 3 (2006): 138-153.

Kaiser, Silke; Mikos, Lothar; Töpper, Claudia. «Orientierung durch Unterhaltung zwischen Fakt und Fiktion. Kinder und die Nutzung des Kinderfernsehens.» Empirische Unterhaltungsforschung: Studien zu Rezeption und Wirkung von medialer Unterhaltung. Hrsg. v. Holger Schramm, Werner Wirth und Helena Bilandzic. München: Reinhard Fischer, 2006. 47-65. 
Kuhlen, Rainer. Wissen als Eigentum? Wie kann der freie Zugang zu den Ressourcen des Wissens in globalen Informationsräumen gesichert werden. Schriftliche Fassung des Einleitungsvortrag der Tagung Wem gehört das Wissen? URL: http://www.bildung2010. de/literatur/kuhlen.pdf.

Töpper, Claudia; Mikos, Lothar. "Chancen und Grenzen medienvermittelter politischer Bildung für bildungsbenachteiligte Jugendliche.» Diskurs Kindheits- und Jugendforschung, 1. Jg., 3 (2006): 403-416. 